\title{
Synthesis of an Epoxy Resin Curing Agent Containing Maleimide Group
}

\author{
Guo Tao ${ }^{1, a^{*}}$, Man Xiao ${ }^{1, b}$, Ziran Chen ${ }^{2, c}$, Youhui Xu ${ }^{2, d}$ \\ ${ }^{1}$ College of Chemistry and Life Science of Chengdu Normal University, Chengdu, Sichuan 611130, \\ P. R. China; \\ 2 Sichuan Vocational and Technical College, Suining, Sichuan 629000, P. R. China;

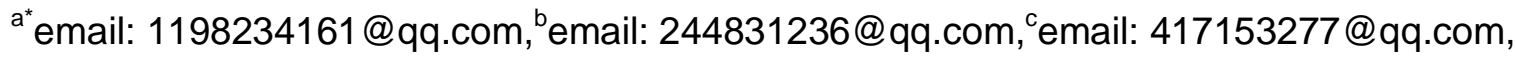 \\ demail: 594760501@qq.com
}

\begin{abstract}
Keywords: maleimide; epichlorohydrin; epoxy resin; curing agent.
Abstract. A novel N, N-(4-amino phenyl maleimide) diglycidyl amine (APMDGA) was synthesized from maleic anhydride, 4-nitroaniline, and epichlorohydrin by a few-step reactions via intermediate products N-(4-nitrophenyl)maleimide and N-(4-aminophenyl)maleimide. The chemical structure of APMDGA is confirmed by $1 \mathrm{H}-\mathrm{NMR}$, FT-IR, and EA. The synthesis conditions have also been investigated. The final product APMDGA shows a yellow solid, and the yield is $48.6 \%$.
\end{abstract}

\section{Introduction}

Epoxy resins have excellent dielectric properties, small product size shrinkage, good stability, strong adhesion to both metallic and non-metallic materials, high hardness, good flexibility, and excellent stability in many solvents and alkali solutions. They have been used as adhesives, coatings, and lamination materials in a wide range of industrial applications such as electronics, mechanics, construction, and aerospace. Epoxy curing agents are used to crosslink epoxy resins and form a three-dimensional network structure, providing the cured epoxy excellent performance. Therefore, the development of new curing agent for epoxy resin is of the same practical importance as the development of new epoxy resin.

To date, the leading epoxy curing agent is amine, followed by acid anhydride, "semi-inorganic polymer" curing agent containing $\mathrm{B}, \mathrm{Si}, \mathrm{P}, \mathrm{F}$, and $\mathrm{Mg}$, modified phenol and mercaptan curing agent, and thiol functionalized block copolymer curing agent. For example, Wang et al. ${ }^{[1]}$ prepared a curing agent BAOP using dichlorophenylphosphine and p-nitrophenol, which is ease of synthesis and has good storage stability, low toxicity, high electrical performance, and low cost. Due to its high phosphorus content, BAOP has excellent flame retardancy and thermal stability, and it can be used as environmentally friendly microelectronics packaging material. $\mathrm{Yu}$ et al. ${ }^{[2]}$ synthesized 3,3',4,4'-diaminodiphenylmethane from 4,4'-Diaminodiphenyl methane. This curing agent has excellent adhesive strength and water resistance. Zhang et al. ${ }^{[3]}$ synthesized 4, 4- glycidyl amino phenyl ether ketone benzene curing agent using 4-Aminophenol, DMF, and toluene, which has good moisture resistance and thermal performance.

In this paper, a novel epoxy curing agent, $\mathrm{N}, \mathrm{N}$ - (4- amino phenyl maleimide) diglycidyl amine (APMDGA), is synthesized from maleic anhydride, 4-nitroaniline, and epichlorohydrin by a few-step reactions via intermediate products $\mathrm{N}$-(4-nitrophenyl)maleimide and $\mathrm{N}$-(4-aminophenyl )maleimide. The primary aim of this work is to enhance film-forming ability and performance of the epoxy resin by the introduction of the functional groups on the curing agent. Specifically, the double bond on the maleimide ring of the curing agent opens to crosslink the epoxy system when the film is formed, which brings about better stability to the film because other curing agents often lead to film with high reaction activity, i.e., the functional groups on the chain ends still remain active even after the curing reaction. As a result, the performance of the epoxy such as hardness and chemical stability will be improved. In addition, this new epoxy curing agent can also be used as a modifying agent for other polymers, i.e., the introduction of the $\mathrm{N}, \mathrm{N}$ - (4- amino phenyl maleimide) functionality results in low porosity and high temperature resistance of the polymeric material. 


\section{Experimental Section}

Materials:All reagents and solvents were in AR grade and were purchased from commercial sources. 4-nitroaniline was obtained from Shanghai Chemical Reagent Company, epichlorohydrin, cupric sulfate, maleic anhydride, magnesium sulfate anhydrous, and sodium carbonate anhydrous were obtained from Tianjin Bodi Chemical Co. Ltd. Toluene was obtained from Chongqing Beibei Chemical Reagent Factory. Hydroquinone, ethyl acetate, N, N- dimethylformamide (DMF), 4-toluenesulfonic acid, ethanol anhydrous, and sodium hydroxide were purchased from Chengdu Kelong Chemical Reagent Factory. Hydrochloric acid was from Guangdong Shantou Xilong Chemical Factory. Tin(II) chloride dihydrate was obtained from Tianjin Kermel Chemical Reagent Co. Ltd. Benzyl triethyl ammonium chloride was obtained from Chengdu Best Reagent Co. Ltd.

\section{Synthesis of N-(4-nitrophenyl)maleimide (NPMI)}

N-(4-nitrophenyl)maleimide(NPMI) was synthesized from p-nitroaniline and maleic anhydride by the following procedure as shown in Figure 1. A four-neck round bottom flask equipped with a mechanical stirrer, a condenser, and a Dean-Stark receiver, was charged with maleic anhydride (14.8 g), DMF $(125 \mathrm{~mL})$, and toluene $(200 \mathrm{~mL})$. The mixture was stirred for $10 \mathrm{~min}$ at room temperature to ensure complete dissolution of maleic anhydride. The flask was then cooled down to $<10{ }^{\circ} \mathrm{C}$ in an ice bath. To this mixture, 4-nitroaniline (17.35g) was added dropwise at a rate of $30 \mathrm{~min} / \mathrm{mL}$, and $0.05 \mathrm{~g}$ of hydroquinone was added as inhibitor. After the completion of the addition, the reaction was left for $12 \mathrm{~h}$ at room temperature under nitrogen while a yellow white slurry was formed.

The second stage of synthesis was accomplished via a cyclization reaction. $0.65 \mathrm{~g}$ of 4-toluenesulfonic acid and $0.065 \mathrm{~g}$ of cupric sulfate were added to the flask, heated up to 140 to 150 ${ }^{\circ} \mathrm{C}$ under nitrogen, and left to stir until no more water droplets appear in the distillate. The reactant turned into a brown-yellow transparent liquid. After cooling down the mixture to $60{ }^{\circ} \mathrm{C}$, a $6 \%$ aqueous $\mathrm{NaHCO}_{3}$ solution was added to neutralize the mixture, and then the mixture was allowed to stand for $24 \mathrm{~h}$ to separate into two layers. The top layer was collected and dropped to $500 \mathrm{~mL}$ of cold deionized water with continuous stirring. Yellow solid precipitated out in water. The precipitate was filtered with a Büchner funnel, washed with deionized water, then vacuum-dried at $70^{\circ} \mathrm{C}$ for $2 \mathrm{~h}$. The final product, a light yellow solid, was obtained. The yield was $85 \%$.

\section{Synthesis of N-(4-aminophenyl)maleimide (APMI)}

A three-neck round bottom flask, under nitrogen, equipped with a mechanical stirrer and a condenser, was charged with $10.0 \mathrm{~g}$ of NPMI, $51.9 \mathrm{~g}$ of Tin(II) chloride dehydrate, and $153 \mathrm{~mL}$ of hydrochloric acid $(6 \mathrm{~mol} / \mathrm{L})$. The reactant was left under stirring for $12 \mathrm{~h}$ at $60{ }^{\circ} \mathrm{C}$, then cooled down to room temperature, and transferred to a $500 \mathrm{~mL}$ beaker. $\mathrm{Na}_{2} \mathrm{CO}_{3}$ was added to neutralize the mixture, followed by extracting with ethyl acetate $(900 \mathrm{~mL})$ three times. The extract solution was collected and dried with magnesium sulfate anhydrous, filtrated, and evaporated in vacuo on a rotary evaporator at $45^{\circ} \mathrm{C}$ while a light yellow solid $(5.20 \mathrm{~g})$ was obtained. The yield of APMI was $50.2 \%$.

\section{Synthesis of N, N- (4- amino phenyl maleimide) diglycidyl amine (APMDGA)}
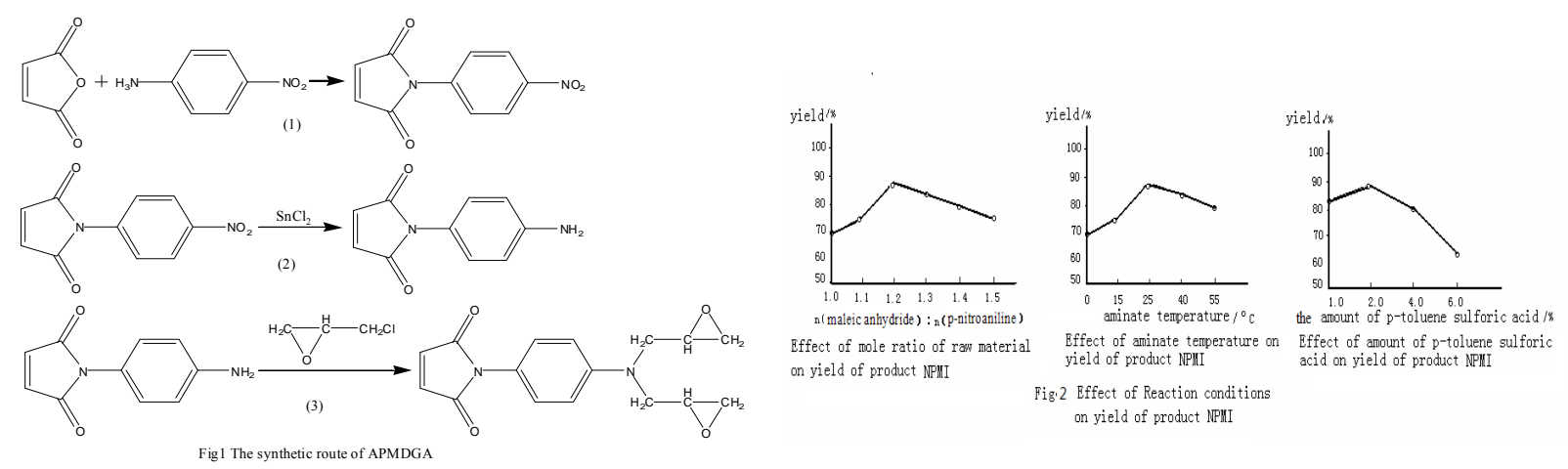

APMI $(6.0 \mathrm{~g})$ and benzyl triethyl ammonium chloride $(0.06 \mathrm{~g})$ were dissolved in $200 \mathrm{~mL}$ of ethanol anhydrous. The solution was heated up to $65{ }^{\circ} \mathrm{C}$ under nitrogen, to which the mixture of epichlorohydrin $(5.9 \mathrm{~g})$ and ethanol anhydrous $(100 \mathrm{~mL})$ was added. The addition process was 
completed within $1 \mathrm{~h}$. The mixture was cooled down to $55^{\circ} \mathrm{C}$, and the reaction was left for $5 \mathrm{~h}$, After completion, the mixture was cooled down to $50{ }^{\circ} \mathrm{C}$ and to which $15.5 \mathrm{~g}$ of $20 \% \mathrm{NaOH}$ solution was added, and left to react for another $3 \mathrm{~h}$ at $50{ }^{\circ} \mathrm{C}$.

The reactant was added dropwise to $1000 \mathrm{~mL}$ of deionized water, followed by adding hydrochloric acid to neutrality. The precipitate was filtered and extracted two times with $1000 \mathrm{~mL}$ of ethyl acetate, then dried with magnesium sulfate anhydrous, and evaporated in vacuo on a rotary evaporator at 45 ${ }^{\circ} \mathrm{C}$. The final product, APMDGA solid $(4.65 \mathrm{~g})$ was obtained. The yield of APMDGA was $48.6 \%$.

Identification of Product:Proton nuclear magnetic resonance( ${ }^{1} \mathrm{H}-\mathrm{NMR}$ )spectra were performed by a Bruker Advance-400 MHz spectrometer, using DMSO-d6 as a solvent and tetramethylsilane as the internal standard. Fourier transform infrared spectroscopy ( FT-IR ) were recorded with a Nicolet FTIR-560 spectrometer by the $\mathrm{KBr}$ salt slice method. Elementary analyses were performed using Italy Euro EA 3000 from Euro Vector S.P.A. Binocular stereo micromelting point apparatus were from Beijing Taike instrument company.

\section{Characterization of NPMI monomers}

Elemental analysis (molecular formula $\mathrm{C}_{10} \mathrm{H}_{6} \mathrm{~N}_{2} \mathrm{O}_{4}$ ): $\mathrm{C} 56.65$ (55.74), H3.89 (4.20), N12.01 (13.45). The experimental results are in brackets, in accordance with the theoretical values. The melting temperature of NPMI is $175^{\circ} \mathrm{C}$.

FT-IR was performed in order to further confirm NPMI structure. The characteristic absorption at $3104 \mathrm{~cm}^{-1}$ corresponds to the $\mathrm{C}=\mathrm{C}$ stretching of the benzene ring. The stretching vibration peaks observed at 1523 and $1349 \mathrm{~cm}^{-1}$ correspond to $\mathrm{N}=\mathrm{O}$. A characteristic absorption peak observed at $1719 \mathrm{~cm}^{-1}$ corresponds to $\mathrm{C}=\mathrm{O}$ stretching in the imide structure.

The shifts and splitting patterns of ${ }^{1} \mathrm{H}-\mathrm{NMR}$ (DMSO-d6) spectra of the NPMI monomer are as follows: $\delta 7.68-7.71(4 \mathrm{H}, \mathrm{Ph}-\mathrm{H}), \delta 7.26(2 \mathrm{H},-\mathrm{CH}=\mathrm{CH}-)$.

The ${ }^{1} \mathrm{H}-\mathrm{NMR}$, FT-IR, and EA results confirm the structure of the NPMI monomer.

\section{Characterization of APMI monomers}

Elemental analysis (molecular formula $\mathrm{C}_{10} \mathrm{H}_{8} \mathrm{~N}_{2} \mathrm{O}_{2}$ ): C65.01 (64.10) , $\mathrm{H} 5.46$ (6.38) , N13.78 (14.66). The experimental results are in brackets, in agreement with the theoretical values. The melting temperature of APMI is $134{ }^{\circ} \mathrm{C}$.

FT-IR $\left(\mathrm{KBr}, \mathrm{cm}^{-1}\right)$ : The characteristic absorption at $3007 \mathrm{~cm}^{-1}$ corresponds to the $\mathrm{C}=\mathrm{C}$ stretching of the benzene ring. The observed peak at $3374 \mathrm{~cm}^{-1}$ corresponds to the stretching vibration peak of $\mathrm{N}-\mathrm{H}$. A characteristic absorption in the imide structure is at $1516 \mathrm{~cm}^{-1}$ which corresponds to $\mathrm{C}=\mathrm{O}$ stretching.

${ }^{1} \mathrm{H}-\mathrm{NMR}$ (DMSO, d): $84.171(2 \mathrm{H}, \mathrm{N}-\mathrm{H}), \delta 6.573-6.595$ and 6.816-6.838(4H, Ph-H), $\delta 6.344(2 \mathrm{H}$, $-\mathrm{CH}=\mathrm{CH}-$ ).

The ${ }^{1} \mathrm{H}-\mathrm{NMR}$, FT-IR, and EA results confirm the structure of the APMI monomer.

\section{Characterization of APMDGA}

FT-IR $\left(\mathrm{KBr}, \mathrm{cm}^{-1}\right)$ : The characteristic absorption peaks at $2953 \mathrm{~cm}^{-1}$ and $2920 \mathrm{~cm}^{-1}$ correspond to the $\mathrm{C}=\mathrm{C}$ stretching of the benzene ring. The absorption peak observed at $2851 \mathrm{~cm}^{-1}$ corresponds to $=\mathrm{C}-\mathrm{H}$ stretching. A characteristic absorption peak at $1519 \mathrm{~cm}^{-1}$ corresponds to the $\mathrm{C}=\mathrm{O}$ stretching in the imide structure. The peaks at $1233 \mathrm{~cm}^{-1}$ and $1188 \mathrm{~cm}^{-1}$ are the asymmetric stretching of the C-O-C group. A characteristic absorption of epoxy structure is at $972 \mathrm{~cm}^{-1}$. A characteristic absorption peak at $1447 \mathrm{~cm}^{-1}$ corresponds to the $\mathrm{C}=\mathrm{C}$ stretching in the imide structure. The observed peak at $1383 \mathrm{~cm}^{-1}$ stands for the $\mathrm{C}-\mathrm{N}-\mathrm{C}$ stretching modes.

${ }^{1} \mathrm{H}-\mathrm{NMR}(\mathrm{DMSO}, \mathrm{d}): \delta 6.853-7.993(6 \mathrm{H}, \mathrm{Ph}-\mathrm{H},-\mathrm{CH}=\mathrm{CH}-), \delta 3.079-3.611(6 \mathrm{H}$,$) ). \delta 2.541-2.733$ $(4 \mathrm{H},-\mathrm{CH} 2-)$,

Elemental analysis (molecular formula $\mathrm{C}_{16} \mathrm{H}_{16} \mathrm{~N}_{2} \mathrm{O}_{4}$ ): $\mathrm{C} 63.99$ (63.05), $\mathrm{H} 5.37$ (5.92) , N 9.33 (9.46), The experimental results are in brackets, in agreement with the theoretical values.

The ${ }^{1} \mathrm{H}-\mathrm{NMR}$, FT-IR, and EA results confirm the structure of APMDGA. 


\section{Results and Discussion}

\section{The synthesized path of APMDGA}

The synthetic route of APMDGA is shown in Figure 1.

\section{Synthesis of N-(4-nitrophenyl)maleimide (NPMI)}

The common methods of synthesizing maleimide include one step, azeotropic distillation, acetic anhydride, phosgene, and esterify ${ }^{[4-6]}$. The most common way is by the reaction of amine with maleic anhydride, via intermediate maleamic acid, then through cyclization, dehydration, and imidization. These methods can be divided into two categories, one using desiccant and the other using catalyst. The first desiccant method is to use excessive or equal amount of desiccant to conduct dehydrating imidization, which leads to high yield. However, this method requires large amount of desiccant, which increases production cost. Furthermore, the product is difficult to separate, which also makes it not suitable for industrial process scale-up. The second catalyst method does not require desiccant; however, if the catalyst has been chosen inappropriate, it will bring about side reactions that cause the yield to decrease ${ }^{[7]}$.

In this work, 4-nitrophenyl maleimide (NPMI) is synthesized with a two-step method from maleic anhydride and 4-nitroaniline in the presence of toluene and DMF, using 4-toluenesulfonic acid as main catalyst and cupric sulfate as second catalyst, with hydroguinol as polymerization inhibitor. The affecting factors for the synthesis were also studied, such as mole ratio of reactants, catalyst amount and reaction temperature. The experimental results are shown in Fig 2.

The optimal reaction conditions for the two-step method are as follows: the molar ratio of maleic anhydride and p-nitroaniline is $1.2: 1$; the amination temperature is 20 to $30{ }^{\circ} \mathrm{C}$; the amount of p-toluene sulfonic acid is 1 to 2 percent of the reactant total mass; the amount of hydroquinone is 0.1 percent of reactant total mass; the amount of cupric sulfate is 0.1 to 0.5 percent of reactant total mass; the cyclization temperature is 145 to $150{ }^{\circ} \mathrm{C}$; the volume ratio of toluene and DMF is $10: 1$, and the cyclization time is more than $6 \mathrm{~h}$. The yield of product is better than $85 \%$.

\section{The synthesized of N-(4-aminophenyl )maleimide (APMI)}

The common methods to deoxidize aromatic functionalized nitrogen-containing compounds into amino-compounds include catalytic hydrogenation, hydrazine hydrate deoxidization, metal deoxidization, electrochemistry deoxidization, etc. ${ }^{[8-13]}$ In this work, APMI is synthesized by a two-step method from NPMI, using Tin(II) chloride dihydrate and hydrochloric acid as catalysts. Using this method, the reaction conditions can be controlled easily, and the yield of the product is more than $50 \%$. The effects of controlling factors such as reaction temperature, catalyst, acidity, and extraction times were investigated as well.

Based on the preliminary results, we investigate the key factors affecting the yield of APMI the reactions such as the amount of reducing agent (A), the amount of hydrochloric acid (B), reaction temperature (C), and the extraction times (D). The optimal conditions were confirmed by $\mathrm{L}_{9}\left(3^{4}\right)$ orthogonal design as shown in Table 1.

\begin{tabular}{|c|c|c|c|c|}
\hline Level & $\begin{array}{l}\text { the amount } \\
\text { of reducer } \\
\text { (A) (mol) }\end{array}$ & $\begin{array}{l}\text { the amount of } \\
\text { hydrochloric acid } \\
\text { (B) (mol) }\end{array}$ & $\begin{array}{l}\text { the reaction } \\
\text { temperature } \\
(\mathrm{C})\left({ }^{\circ} \mathrm{C}\right)\end{array}$ & $\begin{array}{l}\text { the extract } \\
\text { times } \\
\text { (D) }\end{array}$ \\
\hline 1 & 0.184 & 0.69 & 40 & 5 \\
\hline 2 & 0.23 & 0.92 & 60 & 6 \\
\hline 3 & 0.276 & 1.15 & 80 & 7 \\
\hline
\end{tabular}
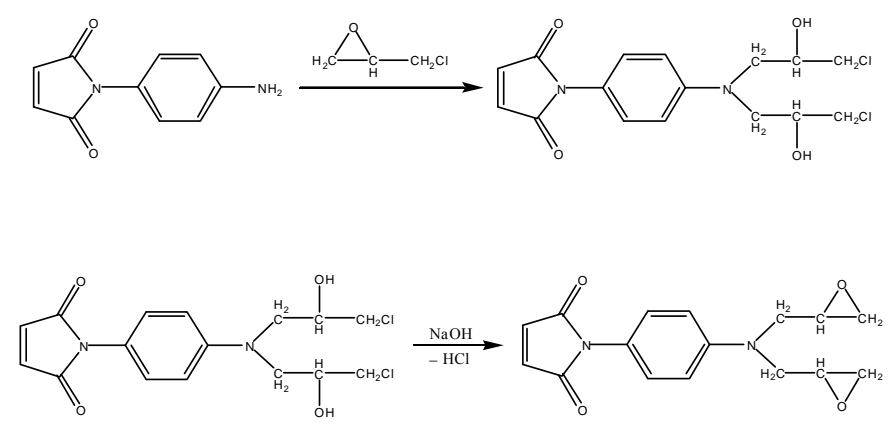

Fig3 The reaction of the amine and epichlorohydrin

The amount of NPMI is $10.0 \mathrm{~g}(0.046 \mathrm{~mol})$. The optimal conditions obtained from the orthogonal experiment results are as follows. The amount of Tin(II) chloride dihydrate reducing agent is $51.9 \mathrm{~g}$ $(0.23 \mathrm{~mol})$; the amount of hydrochloric acid is $153 \mathrm{~mL}(0.92 \mathrm{~mol})$; the reaction temperature ia $60{ }^{\circ} \mathrm{C}$, the extraction times are six. Three repeated experimental results show that the yield of APMI are $50.2 \%, 50.5 \%, 50.3 \%$, indicating good reproducibility. 


\section{The synthesized of $\mathbf{N}, \mathrm{N}$ - (4-amino phenyl maleimide) diglycidyl amine (APMDGA)}

To date, there are two main methods of synthesizing glycidyl amine, i.e., one-step and two-step method $^{[14-18]}$. For the one-step method, the reaction of ring opening addition and dehydrochlorination occur at the same time under the presence of concentrated alkali, using trimethylamine and epichlorohydrin as raw materials. The production process requires long reaction time, is easy to form a polymer, and the yield is low. Therefore, it is difficult to scale up. The two-step method also utilizes amine and epichlorohydrin as raw materials. According to the phase transfer catalysis technology from Makosza, Starks, and Brad strom in late 1960s, the first step reaction is formation of intermediate product amino chloro propanol, then form glycidyl amine in the presence of concentrated alkali. As compared to the one-step method, the two-step method has the advantages such as mild reaction conditions, ease of operation, high selectivity, and high yield. However, the feed ratio $\mathrm{n}$ (Epichlorohydrin) to $\mathrm{n}$ (amine) is large, which requires more epichlorohydrin amount; furthermore, the cost of glycidyl amine is high, so production is not easy to scale up.

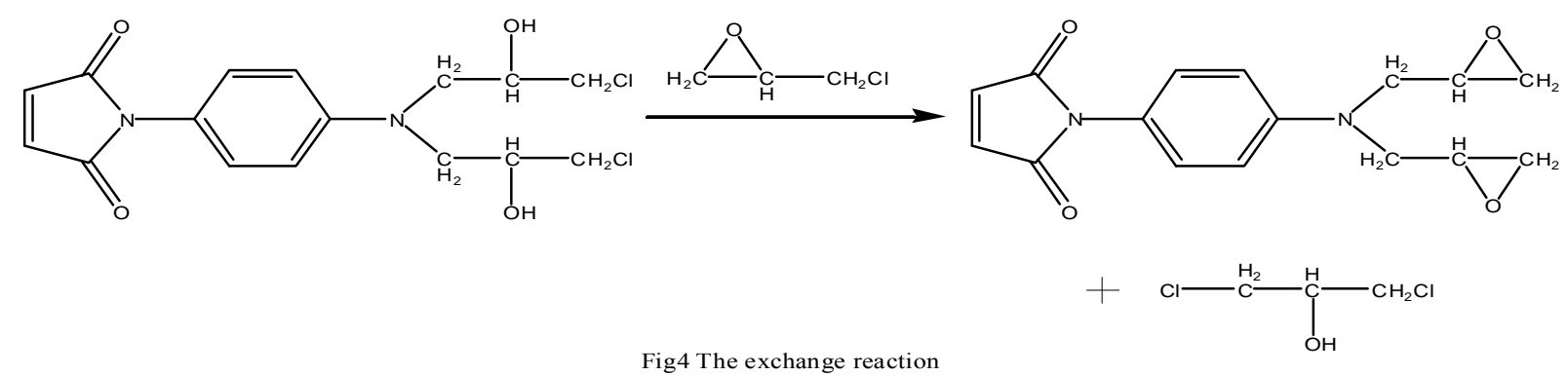

In this work, we employ the two-step method to synthesize glycidyl amine, using benzyl triethyl ammonium chloride as the phase transfer catalyst. Benzyl triethyl ammonium chloride has good dissolution ability in both aqueous phase and organic phase, i.e., cations and anions of quaternary ammonium salt form ion pairs in the aqueous phase, anions could transfer from the aqueous phase to the organic phase. In the organic phase, anions could not provide solvation. Additionally, due to the large size of quaternary ammonium cation, the distance between cations and anions is large, resulting in weak interactions, which accelerates the reaction rate.

The first step is to form the intermediates amino chloro propanol from amine and epichlorohydrin, then to produce glycidyl amine in the presence of sodium hydroxide, as shown in Figure 3. The addition reaction rate of amine and epichlorohydrin is often very slow; however, in the presence of a small amount of phase transfer catalyst and second catalyst, the reaction rate accelerates while the intermediate product amino chloro propanol is produced. Then amino chloro propanol form glycidyl amine by exchange with excess epichlorohydrin in the system, as shown in Figure 4. The conversion of this reaction depends on the structure of amine.

\section{Conclusion}

A novel N, N- (4- amino phenyl maleimide) diglycidyl amine (APMDGA) was synthesized from maleic anhydride, $\mathrm{p}$-nitroaniline, epichlorohydrin by a few-step reactions via intermediate products $\mathrm{N}$-(4-nitrophenyl)maleimide and N-(4-aminophenyl)maleimide. The chemical structure of APMDGA was confirmed by $1 \mathrm{H}-\mathrm{NMR}$, FT-IR, and EA. The synthesis conditions have also been investigated. The final product APMDGA shows a yellow solid, and the yield is $48.6 \%$. The application of APMDGA is under investigation.

\section{Acknowledgements}

This work was financially supported by Sichuan Province Key Technology Support Program (2011GZ0247) and Sichuan Province Education Office Natural Science Foundation under grant 14ZA0300 and 14ZA0371. 


\section{References}

[1]Wang Hong-juan, Wang Xin-long, Wang Qing-hua,Synthesis and application of the phosphorous curing agents of epoxy resin,Insulating material. 2(2005)4-7 .

[2]Yu Xin-hai,Guo Xiang, Chen Ji-wei, Liu Wan-zhang, Hu Bin,Preparation of novel solvent-free epoxy adhesives with high-temperature resistance,Adhesion.1(2014)33-39 .

[3]Zhang Chun-ling,Sun Guo-en, Zhang Li,Curing properties of the novel curing agent of epoxy resin, Journal of Jilin Univ ersity ( Engineering and Technolo gy Edition).37(2007)353-356 .

[4]T.Agag,T.Takeichi,Synthesis and characterization of novel benzoxazine monomers containing allyl groups and their high performance thermosets,Macromolecules.36( 2003)6010-6017.

[5] H.Ishida,D.P.Sanders, Improved thermal and mechanical properties of polybenzoxazines based on alkyl-substituted aromatic amines, J Polym Sci B: Polym Phys.38( 2000)3289-3301.

[6] A.Chernykh, J.P.Liu,H.Ishida,Synthesis and properties of a new crosslinkable polymer containing benzoxazine moiety in the main chain,Polymer.47( 2006)7664-7669.

[7]T.Takeichi,T.Kano,T.Agag,Synthesis and thermal cure of high molecular weight polybenzoxazine precursors and the properties of the thermosets,Polymer.46( 2005)12172-12180.

[8] Y.L.Liu ,J.M.Yu,C.I.Chou,Preparation and properties of novel benzoxazine and polybenzoxazine with maleimide groups,J. Polym Sci A: Polym Chem.42( 2004)5954-5963.

[9] H.Ishida,Y.H.Lee,Infrared and thermal analyses of polybenzoxazine and polycarbonate blends, J. Appl. Polvm. Sci.81(2001)1021 1034.

[10]K.Hemvichian,A.Laobuthee,S.Chirachanchai,H.Ishida,Thermal decomposition processes in Polybenzoxazine model dimers investigated by TGA-FTIR and GC-MS,Polym Degrad Stab. 76(2002)1-15.

[11]H.Ishida,S.Ohba,Synthesis and characterization of maleimide and norbornene functionalized benzoxazines,Polymer.46(2005)5588-5595.

[12]Yan Tang, Zhao Qinglai, Ke Zhou,Synthesis of a benzoxazine monomer containing maleimide and allyloxy groups,Chinese Chemical Letters.18(2007)973 976.

[13]Cui Jian,Reserch advance of N-phenyl maleimide synthesis and its application,Modern plastics processing and applications. 13(2001)53-55.

[14]Li Zhi-fu,Studies on the preparation of N-phenyl maleimide,Shandong chemical industry. 29(2000)13-14.

[15]Xu Bin, Jiang Yong,Study on the synthesis of N-phenyl maleimide,Modern plastics processing and applications.15(2003)13-16.

[16]Meng Ming-yang, Ma Ying, Tan Li-zhe,Technology and equipment for hydrogenation reduction, Fine and specialty chemicals.24(2004)311-314.

[17]D.J.Liaw,B.Y.Liaw,C.W.Yu,Synthesis and characterization of new organosoluble polymides based on flexible diamine,Polymer.42(2001)5175-5179.

[18]C.S.Wang,T.S.Leu,Synthesis and characterization of polyimides containing naphthalene pendant group and flexible ether linkages,Polymer.41(2000)3581-3591. 